\author{
ACTA MYCOLOGICA \\ Vol. 43 (2): 161-165 \\ 2008
}

\title{
Three species of the genus Agaricus new to Poland
}

\author{
JANUSZ ŁUSZCZYŃSKI \\ Department of Botany, Institute of Biology, Jan Kochanowski University \\ Świętokrzyska 15, PL-25-406 Kielce, jluszcz@ujk.kielce.pl
}

Łuszczyński J.: Three species of the genus Agaricus new to Poland. Acta Mycol. 43 (1): 161-165, 2008.

The paper presents informations on Agaricus excellens (F. H. Møller) F. H. Møller, A. maleolens F. H. Møller and A. romagnesii Wasser, three new species for Poland.

Key words: Agaricus excellens, A. maleolens, A. romagnesii, Góry Świętokrzyskie Mts., Kielce, Poland

\section{INTRODUCTION}

Fruitbodies of fungi belonging to the genus Agaricus are characterised by a white or yellow pileus and free gills with a regular trama when young, later becoming irregular. Spores are globose, oval or elliptical and the spore print is purple-brown to black-brown. The spore wall is smooth, often thick and indistinctly pseudoamyloid. Cheilocystidia either occur or are absent. The shape, size and attachment of the ring are important diagnostic features of the genus. Much attention is also paid to the reaction of the flesh in aniline, concentrated $\mathrm{HNO}_{3}$, strong $\mathrm{NaOH}$ or $\mathrm{KOH}$ and to the discolouration of the cuticle or the flesh on exposure to air when the fruitbody is rubbed or broken.

Despite large fruitbodies, few fungi of the genus Agaricus are known in Poland and were until recently represented merely by 28 species (Wojewoda 2003). In contrast, ca. 55 species are known in Germany (Krieglsteiner 1991), Poland's neighbour where climatic and habitat conditions are similar. Nearly all species of Agaricus known to grow in Europe occur there (Horak 2005). In Poland, only four species: A. arvensis, $A$. campestris, $A$. silvaticus and $A$. silvicola, can be regarded as common, and further six: $A$. augustus, $A$. bitorquis, $A$. comptulus, $A$. niveolutescens, $A$. semotus and $A$. xanthodermus as quite frequent with the number of known localities ranging from 10 and 20 . The other 18 species are known only from few or single localities. Often cosmopolitan, species of this genus occur in a variety of habitats: natural (forests), seminatural non-forest environments (meadows, shrubs and pastures), 
as well as segetal (fields, gardens) and ruderal (parks, lawns); usually, however, in sites rich in humus.

Three species of the genus Agaricus, $A$. excellens, $A$. maleolens and $A$. romagnesii, new to the Polish mycobiota were found during mycocoenobiotic studies in the Góry Świętokrzyskie Mts. (Łuszczyński 2007, 2008). The collected material was deposited in the herbarium of the Department of Botany, Institute of Biology, Jan Kochanowski University, Kielce.

\section{SPECIES DESCRIPTION}

Agaricus excellens (F. H. Møller) F. H. Møller, Friesia 4: 204 (1952).

Syn.: Psalliota augusta Fries sensu Ricken, Die Blätterpilze, p. 235 (1915). - Agaricus urinascens (Jul. Schäff. \& F.H. Møller) Singer, Lilloa 22: 431 (1951). - Psalliota excellens F.H. Møller, Friesia 4: 178 (1952). - Agaricus macrosporus subsp. excellens (F.H. Møller) Bohus, Ann. Hist.-Nat. Mus. Natl. Hung. 70: 105 (1978). - Agaricus macrosporus var. excellens (F.H. Møller) Vasas, Ann. Hist.-Nat. Mus. Natl. Hung. 82: 41 (1990). - Agaricus urinascens var. excellens (F.H. Møller) Nauta, Persoonia 17(3): 462 (2001) [2000].

ICONOGRAPHY: Møller 1952. Friesia 4, Fig. 29, pl. XXXIII. - Essette. 1964. Les Psalliotes, Tab. 44. - Wasser 1980. Flora Fungorum RSS Ucrainicae, Figs. 68-69. - Phillips 1981. Mushrooms, p. 167. - Cappelli 1984. Agaricus L.: Fr. ss. Karsten (Psalliota Fr.), text Fig. $29+$ icon. Fig. 41. - Michael et al. 1985. Handbuch für Pilzfreunde 4, Tab. 15. - Wasser 1985. Agarikovyje griby, Tab. 4(6). - Hagara 1987. Atlas húb, p. 324.

Description. Species of the section Flavescentes. Pileus spherical convex, at first pure white, finely squamulose along the margin in young fruitbodies. Cuticle discolours yellow, chrome-yellow when rubbed. Pileus ochre-yellow in older fruitbodies, up to $15 \mathrm{~cm}$. in diam, densely covered in fine squamules. Stipe white, clavate, up to $12 \mathrm{~cm}$ long, $3 \mathrm{~cm}$ in diam., squamulose in lower part, glabrous in upper part. Ring strong, pendant, squamulose on the outside. Flesh white, becoming pink on cutting, smell slightly amygdaline. Spores 9.45-10.8 × 5.4-6.7 $\mu \mathrm{m}$. Cheilocystidia numerous, oval, clavate, 15-30 × 5.4-14.8 $\mu \mathrm{m}$ (Fig. 1).

Localities. In forests: PeucedanoPinetum and Tilio-Carpinetum, on ground, September-October, locali-
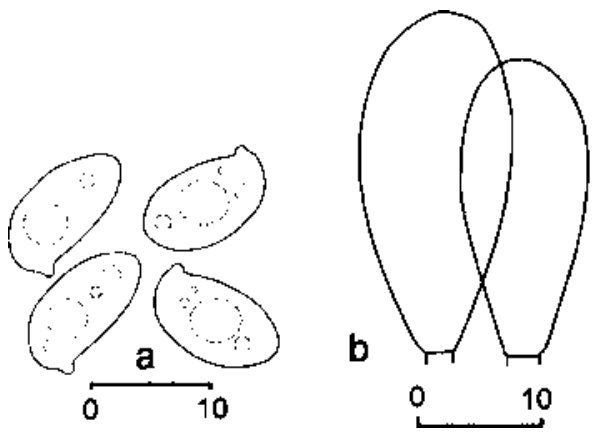

Fig. 1. Agaricus excellens: $\mathrm{a}$ - spores, $\mathrm{b}$ - cystidia.

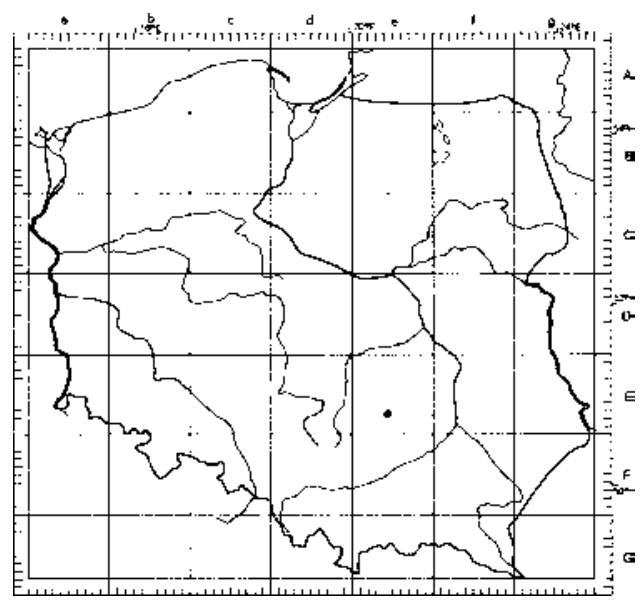

Fig. 2. Localities of Agaricus excellens, $A$. maleolens and $A$. romagnesii in square of the ATPOL net. 
ties: Kielce (Mt. Biesak, Bukówka, Mt. Telegraf) and Dyminy. All localities in the ATPOL square Ee 74 (Fig. 2).

Distribution. The fungus occurs in many European countries, for instance in Austria, Belgium, Bulgaria, the Czech Republic, Denmark, France, Germany, Great Britain, Hungary, Italy, the Netherlands, Norway, Portugal, Slovakia, Slovenia, Switzerland, Ukraine (Bohus 1978; Hansen, Knudsen 1992; Hernández-Crespo 2006; Kreisel 1987; Krieglsteiner 1991; Lacheva 2006; Wasser 1980, 1985). It is also known from Eastern Asia (Krasnoyarsk Krai, Wasser 1985) and Africa from Morocco (Malençon, Bertault 1970).

Agaricus maleolens F. H. Møller, Friesia 4: 203 (1952).

Syn.: Agaricus campestris subsp. bernardii (Quél.) Konrad \& Maubl., Icones 6 (texte): 60 (1937). Agaricus ingratus (F.H. Møller) Pilát, Acta Musei Nationalis Pragae, 7 B, 1 (1951). (Nom. inval.). - Pratella bernardii (Quél.) Quél., Fl. Mycol. France (Paris): 73 (1888). - Psalliota bernardii (Quél.) Quél. [as 'bernardi'], Bull. Soc. Bot. France 25: 288 (1879) [1878]. - Psalliota ingrata F.H. Møller, Friesia 4: 17 (1950), non Agaricus ingratus Fries (= Gymnopus confluens).

ICONOGRAPHY. Møller 1952. Friesia 4, Fig. 5, pl. VIII. - Wasser 1980. Flora Fungorum RSS Ucrainicae, Fig. 62, Tab. IX. - Michael et al. 1985. Handbuch für Pilzfreunde 4, Tab. 9. - Cappelli 1984. Agaricus L.: Fr. ss. Karsten (Psalliota Fr.), text Fig. 3 + icon. Fig. 3. - Wasser 1985. Agarikovyje griby, Tab. 4(1).

DesCRIPTION. Pileus hemispherical convex, glabrous, $5-7 \mathrm{~cm}$ in diam., white-ochre. Margin draped with the remains of the partial veil. Gills thin, crowded, chocolatecoloured. Stipe cylindrical, evenly thick, concolourous with pileus, $6-8 \times 1.8-2 \mathrm{~cm}$. Ring thin, spreading, falls down pulling away from the stipe. Flesh ochrepink on cutting, darkening, darkening particularly quickly in the stipe and becoming brunneous-ochre with a distinctive pink tint. Smell of fresh fish, stronger and unpleasant fishy smell when drying. Spores broadly elliptical, 5.4-6.6 x 4.2-5 $\mu \mathrm{m}$, redbrown, mostly with a drop of fat (Fig.
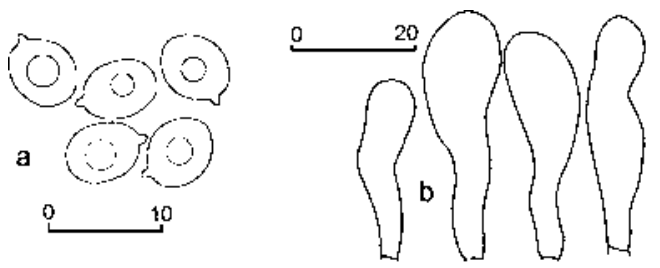

Fig. 3. Agaricus maleolens: a - spores, b - cystidia. 3). Cheilocystidia hyaline, cylindrical, with a blunt hemispherical tip, erect or arching ascendant, 6-8 $\mu \mathrm{m}$ in diam., on gill edges.

LocAlity. In urban area, on lawn, under Populus sp., May, locality: Kielce (Szydłówek), ATPOL square Ee 74.

Distribution. Rare throughout Europe; known from Germany, Switzerland (Krieglsteiner 1991), Denmark, Finland, Sweden (Hansen, Knudsen 1992), Bulgaria (Lacheva 2006), Hungary, Slovakia, the Czech Republic, Spain, Portugal, Italy, the Netherlands, France, Ukraine (Wasser 1985). Also known from Africa from Morocco (Malençon, Bertault 1970).

Agaricus romagnesii Wasser, Ukrainian Botanical Journal 34: 305 (1977).

Syn.: Agaricus radicatus (Vittad.) Romagnesi, Bull. Soc. Mycol. France, 53, p. 129 (1937) (Nom. inval.), non Agaricus radicatus Rehl.: Fries 1821. - Psalliota radicata (Vittad.) Essette, Les Psalliotes, Tab. 22 (1964) (Nom. inval.). - Agaricus bresadolanus Bohus, Ann. Hist.-Nat. Mus. Natl. Hung. 61: 154 (1969). Agaricus bresadolanus Bohus sensu Reid, Fungorum Rariorum Icones Coloratae 6, p. 6 (1972). - Psalliota infida Alessio, Micol. Ital. 4(2): 21 (1975) (Nom. inval.). - Agaricus infidus (Alessio) Bon, Doc. Mycol. 11(44): 28 (1981) (Nom. inval.), non Agaricus infidus Peck 1900.

ICONOGRAPHY. Romagnesii 1937. Bull. Soc. Mycol. France, 53, p. 130. - Essette 1964. Les Psalliotes, Tab. 22. - Bohus 1971. Ann. Hist.-Nat. Mus. Natl. Hung. 63, p. 79. - Reid 1972. Fungorum Rariorum 
Icones Coloratae 6, pl. 42. - Rinaldi et al. 1974. Latlante dei funghi, Fig. 4. - Alessio 1975. Micol. Ital. 4(2), Tab. 11. - Wasser 1977. Ukrainian Botanical Journal 34(3): 85. - Wasser 1980. Flora Fungorum RSS Ucrainicae, Tab. I(1). - Blatto 1982. Atlante fotografico dei funghi, Tab. 31. - Cappelli 1984. Agaricus L.: Fr. ss. Karsten (Psalliota Fr.), text Fig. 40 + icon. Fig. 70. Wasser 1985. Agarikovyje griby, Tab. 1 (3).

Description. Pileus at first white, later darker, beige, covered in light-brown, brown squamules and fibres; spherically convex when young, flattened, centrally depressed, up to ca. $8 \mathrm{~cm}$ in diam. in older specimens. Stipe white, later rusty-yellowing, cylindrical, slightly thickened at the base, contracting into quite thick rhizomor-

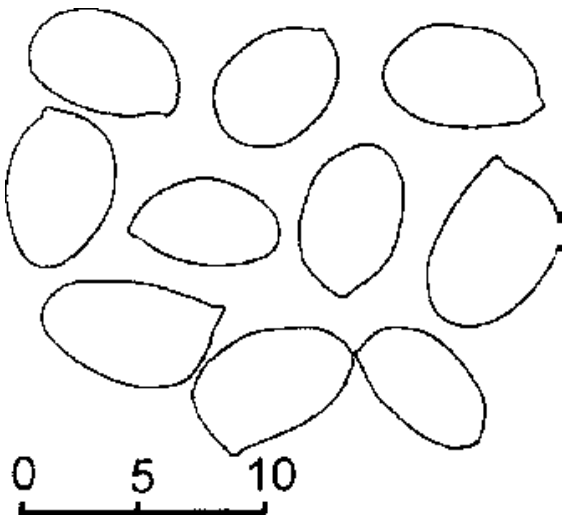

Fig. 4. Agaricus romagnesii: spores.

phic strands, one or more. Ring thin, delicate, not persistent. Gill edges sterile, with quite numerous hyaline, clavate cheilocystidia, 30-40 x 8.1-10.8 $\mu \mathrm{m}$. Spores broadly elliptical, 6.5-8 x 3.5-5 $\mu \mathrm{m}$ (Fig. 4).

Locality. In pastures: Lolio-Cynosuretum, on ground, July, locality: Kielce (Nowy Folwark), ATPOL square Ee 74.

Distribution. In southern and central Europe; known from Slovenia, Germany, France, Bulgaria, the Czech Republic, Slovakia, Ukraine, Hungary, Great Britain, Italy, Spain, Portugal (Hernández-Crespo 2006; Lacheva 2006; Parra 2003). Also known from Asia, Israel, Uzbekistan, Turkmenistan, Tadzhikistan (Wasser 1985), Turkey (Kasiik et al. 2003), Georgia [http://www.cybertruffle.org.uk/ gruzmaps/a/0020960_.htm].

\section{REFERENCES}

Alessio C. L. 1975. Psalliota radicata Vitt. sensu Bres. e P. radicata sensu Essette: nome nuovo per la seconda: Psalliota infida (= Agaricus infidus) sp.n. Micol. Ital. 4(2): 16-22.

Blatto L. 1982. Atlante fotografico dei funghi. Hoepli. Milano.

Bohus G. 1971. Agaricus studies. III. Ann. Hist.-Nat. Mus. Natl. Hung. 70: 105-110.

Bohus G. 1978. Agaricus studies. VIII. Ann. Hist.-Nat. Mus. Natl. Hung. 63: 77-82.

Bohus G. 1990. Agaricus studies. XI (Basidiomycetes, Agaricaceae). Monographical key. Ann. Hist.-Nat. Mus. Natl. Hung. 82: 39-59.

Bon M. 1981. Novitates. Combinaisons et noms nouveaux. Doc. Mycol. 11 (44): 1-28.

Cappelli A. 1984. Agaricus L.: Fr. ss. Karsten (Psalliota Fr.). Fungi Europaei. Libreria editrice Biella Giovanna. Saronno.

Essette H. 1964. Les Psalliotes. Lechevalier. Paris.

Hagara L. 1987. Atlas húb. Vydavatel'stvo Osveta. Martin.

Hansen L., Knudsen H. 1992. (eds). 1992. Nordic Macromycetes. 2. Polyporales, Boletales, Agaricales, Russulales. Nordsvamp. Copenhagen.

Hernández-Crespo J. C. 2006. S I M I L. An on line Information System of the Iberian Fungal Diversity. Royal Botanic Garden Madrid, C.S.I.C. Research Project Flora Micológica Ibérica I-VI (1990-2008). Spanish Ministry of Education and Science. [http://www.rjb.csic.es/fmi/sim.php].

Horak E. 2005. Röhrlinge und Blätterpilze in Europa. Elsevier, Spektrum Akademischer Verl. München.

Kaşik G., Türkoğlu A., Doğan H. H., Őstürk C. 2006. Macrofungi of Yahjalı (Kayseri) Province. Turkish Journal of Botany 27: 453-462.

Konrad P., Maublanc A. 1937. Icones selectae Fungorum, 6. Lechevalier. Paris. 
Kreisel H. (ed.). 1987. Pilzflora der Deutschen Demokratischen Republik. Basidiomycetes (Gallert-, Hutund Bauchpilze). VEB G. Fischer Verl. Jena.

Krieglsteiner G. J. 1991. Verbreitungsatlas der Großpilze Deutschlands (West). 1. Teil b. Verl. Eugen Ulmer. Stuttgart.

Lacheva M. N. 2006. Genus Agaricus L.: Fr. emend. P. Karst. (mushroom) in Bulgaria - taxonomy, ecology, chorology and economical importance. Ph. D. thesis. Agrar. Univ. Plovdiv [http://botanica.hit. bg/doc/AgaricusPhDthesis.pdf].

Łuszczyński J. 2007. Diversity of Basidiomycetes in various ecosystems in Góry Świętokrzyskie Mts. Monogr. Bot. 97: 1-218.

Łuszczyński J. 2008. Basidiomycetes of the Góry Świętokrzyskie Mts. A checklist. Wyd. Uniwersytetu Humanistyczno-Przyrodniczego Jana Kochanowskiego w Kielcach, Kielce (in print).

Malençon G., Bertault R. 1970. Flore des champignons superieurs du Maroc. 1. Faculte des Science. Rabat.

Michael E., Hennig B., Kreisel H. 1985. Handbuch für Pilzfreunde, 4. VEB G. Fischer Verl. Jena.

Møller F. H. 1952. Danish Psalliota species, I-II. Friesia 4: 135-220.

Nauta M. M. 2001 [2000]. Notulae ad floram agaricinam neerlandicam. XXXVII. Notes on Agaricus section Arvenses. Persoonia 17 (3): 457-463.

Parra L. A. 2003. Números 2070-2123. (In:) J. C. Hernández (ed.). Bases corológicas de Flora Micológica Ibérica. Números 2070-2178 Caudernos de Trabajo de Flora Micológica Ibérica 19: 19-104.

Pilát A. 1951. The Bohemian species of the genus Agaricus. Acta Musei Nationalis Pragae, 7 B, 1: 1-142. Phillips R. 1981. Mushrooms. Pan Books Ltd, Londra.

Quélet L. 1878. Quelques espèces nouvelles de champignons (6. suppl.). Bull. Soc. Bot. France 25: 288.

Quélet L. 1888. Flore mycolgique de la France et des pays limitrophes. Octave Doin, Paris.

Reid D. A. 1972. Fungorum Rariorum Icones Coloratae, 6. J. Cramer, Vaduz.

Ricken A. 1915. Die Blätterpilze. Leipzig.

Rinaldi A., Tyndalo V., Pace G. 1974. Latlante dei funghi. Mondadori.

Romagnesi H. 1937. Liste des champignons supérieurs recueillis à Paris. Bull. Soc. Mycol. France 53: 128-131.

Singer R. 1951. The "Agaricales" (mushrooms) in modern taxonomy, Lilloa 22: 1-832.

Wasser S. P. 1977. New and rare species of Agaricaceae Cohn. family. Ukrainian Botanical Journal 34 (3): 305-307.

Wasser S. P. 1980. Agaricaceae Cohn. Flora Fungorum RSS Ucrainicae. Naukova Dumka.Kiev.

Wasser S. P. 1985. Agarikovyje griby SSSR. AN USSR. Naukova Dumka. Kiev.

Wojewoda W. 2003. Checklist of Polish larger Basidiomycetes. (In:) Z. Mirek (ed.). Biodiversity of Poland. 7. W. Szafer Institute of Botany, Polish Academy of Sciences, Kraków.

\section{Gatunki z rodzaju Agaricus nowe dla Polski}

\section{Streszczenie}

Rodzaj Agaricus reprezentowany jest w Polsce przez 28 gatunków. Autor przedstawia trzy nowe dla Polski gatunki z tego rodzaju: $A$. excellens, $A$. maleolens i $A$. romagnesii zebrane podczas długoterminowych badań mikocenologicznych w Górach Świętokrzyskich.

Agaricus excellens został odnaleziony w zbiorowisku Peucedano-Pinetum i Tilio-Carpinetum na ziemi, $A$. maleolens na ziemi pod Populus sp. w strefie miejskiej Kielc, a $A$. romagnesii w zbiorowisku Lolio-Cynosuretum.

Autor podaje szczegółowe opisy morfologiczne owocników, siedlisk występowania i zbioru oraz rozmieszczenie geograficzne w Polsce i Europie. 